\title{
A Generalized Occupation Time Formula For Continuous Semimartingales
}

\author{
Raouf Ghomrasni \\ School of Computational \& Applied Mathematics, \\ University of the Witwatersrand, \\ Private Bag 3, Wits, 2050 Johannesburg, South Africa. \\ E-mail:rghomrasni@cam.wits.ac.za
}

\begin{abstract}
We show that for a wide class of functions $F$ that:$$
\lim _{\varepsilon \downarrow 0} \frac{1}{\varepsilon} \int_{0}^{t}\left\{F\left(s, X_{s}\right)-F\left(s, X_{s}-\varepsilon\right)\right\} d\langle X, X\rangle_{s}=-\int_{0}^{t} \int_{\mathbb{R}} F(s, x) d L_{s}^{x}
$$

where $X_{t}$ is a continuous semi-martingale, $\left(L_{t}^{x}, x \in \mathbb{R}, t \geq 0\right)$ its local time process and $\left(\langle X, X\rangle_{t}, t \geq 0\right)$ its quadratic variation process.
\end{abstract}

Key words and phrases: Continuous semimartingale, local time, occupation time formula.

MSC2000: 60H05, 60J65.

\section{Introduction}

Recently Feng and Zhao [1] define the integral of local time $\int_{0}^{t} \int_{\mathbb{R}} g(s, x) d L_{s}^{x}$ pathwise and then they derived a generalized Itô's formula when $\nabla^{-} F(s, x)$ is only of bounded $p, q$ variation in $(s, x)$. In the case that $g(s, x)=\nabla^{-} F(s, x)$ is of locally bounded variation in $(s, x)$, the integral $\int_{0}^{t} \int_{\mathbb{R}} \nabla^{-} F(s, x) d L_{s}^{x}$ is the Lebesgue-Stieltjes integral. When $g(s, x)=$ $\nabla^{-} F(s, x)$ is of only locally $p, q$-variation, where $p \geq 1, q \geq 1$, and $2 q+1>2 p q$, the integral is a two-parameter rough path integral rather than a Lebesgue-Stieltjes integral.

In section 2, we first study the time-independent case and establish a formula wich in particular unify the expression of local time of a continuous semimartingales defined as

$$
L_{t}^{a}=\lim _{\varepsilon \downarrow 0} \frac{1}{\varepsilon} \int_{0}^{t} 1_{[a, a+\varepsilon[}\left(X_{s}\right) d\langle X, X\rangle_{s}
$$

and the expression of the quadratic variation process in terms of contributions coming from fluctuations in the process that occur in the vicinity of different spatial points $a \in(-\infty, \infty)$ :

$$
\langle X, X\rangle_{t}=\int_{\mathbb{R}} L_{t}^{a} d a
$$

we then deal with the time-dependent case. A recent survey of semimartingales local time and occupation density concepts is given by I. Serot in [2]. 


\section{Main Results}

\subsection{Time independent Case}

Using Lyons-Youngs integration of one parameter $p$-variation, Feng and Zhao [1] defined $\int_{\mathbb{R}} F(x) d_{x} L_{t}^{x}$ as a rough path integral if $F(x)$ is of bounded $p$-variation $(1 \leq p<2)$. They also proved a dominated convergence theorem ([1] Theorem 2.1) for the rough path integral and then extended Meyer's formula to $F(x)$ is of bounded $p$-variation $(1 \leq p<2)$. We shall use their results in order to establish the following theorem.

Theorem 1 Let $F$ be a left continuous function with bounded $p$-variation $(1 \leq p<2)$, we have the following:

$$
\lim _{\varepsilon \downarrow 0} \frac{1}{\varepsilon} \int_{0}^{t}\left\{F\left(X_{s}\right)-F\left(X_{s}+\varepsilon\right)\right\} d\langle X, X\rangle_{s}=-\int_{\mathbb{R}} F(x) d_{x} L_{t}^{x}
$$

Remark 2.1 1. If we take $F(t, x)=1_{(x \leq a)}$ in (2.1) we have the very definition of $L_{t}^{a}$

2. If we take $F(x)=x$ in (2.1) we have $\langle X, X\rangle_{t}=\int_{\mathbb{R}} L_{t}^{x} d x$.

Proof: Let us associate to $F$ the following function:

$$
H_{\varepsilon}(x):=\frac{1}{\varepsilon} \int_{x}^{x+\varepsilon} F(y) d y
$$

On the one hand we have:

$$
H_{\varepsilon}(x):=\frac{1}{\varepsilon} \int_{x}^{x+\varepsilon} F(y) d y \rightarrow \quad F(x) \text { for } \varepsilon \rightarrow 0
$$

On the other hand

$$
\frac{\partial}{\partial x} H_{\varepsilon}(x):=\frac{1}{\varepsilon}\{F(x+\varepsilon)-F(x)\}
$$

We note that the function $H_{\varepsilon}(x)$ in $(2.2)$ is of bounded $p$-variation $(1 \leq p<2)$ for any fixed $\varepsilon>0$. This is may be easily proved by checking the definition of $p$-variation or as communicated to the author by Prof. Lyons:

(i) the property of having finite $p$-variation $(p<2)$ can be expressed in terms of a norm being bounded.

(ii) the property is preserved under translation.

(iii) the ball in any norm is convex.

(iv) the function $H_{\varepsilon}$ is defined as an integral with a convex combination of translates of the original path.

It follows, from Theorem 2.1 in ([1])

$$
\int_{\mathbb{R}} H_{\varepsilon}(x) d_{x} L_{t}^{x} \rightarrow \int_{\mathbb{R}} F(x) d_{x} L_{t}^{x}
$$

and

$$
\int_{\mathbb{R}} H_{\varepsilon}(x) d_{x} L_{t}^{x}=\frac{1}{\varepsilon} \int_{0}^{t}\left\{F\left(X_{s}\right)-F\left(X_{s}+\varepsilon\right)\right\} d\langle X, X\rangle_{s}
$$

We use here Feng-Zhao theorem (2.1) ([1]) 


\subsection{Time-dependent Case}

Theorem 2 Let $F:[0, t] \times \mathbb{R} \rightarrow \mathbb{R}$ be a left continuous, locally bounded with bounded $\gamma$ variation in $x$ uniformly in $s$ and of bounded $p, q$-variation in $(s, x)$, where $1 \leq \gamma<2$ and $p, q \geq 1,2 q+1>2 p q$, Then

$$
\lim _{\varepsilon \downarrow 0} \frac{1}{\varepsilon} \int_{0}^{t}\left\{F\left(s, X_{s}\right)-F\left(s, X_{s}-\varepsilon\right)\right\} d\langle X, X\rangle_{s}=-\int_{0}^{t} \int_{\mathbb{R}} F(s, x) d L_{s}^{x}
$$

and also,

$$
\lim _{\varepsilon \downarrow 0} \frac{1}{2 \varepsilon} \int_{0}^{t}\left\{F\left(s, X_{s}-\varepsilon\right)-F\left(s, X_{s}+\varepsilon\right)\right\} d\langle X, X\rangle_{s}=\int_{0}^{t} \int_{\mathbb{R}} F(s, x) d L_{s}^{x}
$$

Proof: By Remark 4.1 and Theorem 4.2 in Feng and Zhao (Two-parameter $p, q$-variation Paths and Integrations of Local Times): Let us associate to $F$ the following function:

$$
H_{\varepsilon}(t, x):=\frac{1}{\varepsilon} \int_{x}^{x+\varepsilon} F(t, y) d y
$$

On the one hand we have:

$$
H_{\varepsilon}(t, x):=\frac{1}{\varepsilon} \int_{x}^{x+\varepsilon} F(t, y) d y \rightarrow \quad F(t, x) \quad \text { for } \quad \varepsilon \rightarrow 0
$$

On the other hand

$$
\frac{\partial}{\partial x} H_{\varepsilon}(t, x):=\frac{1}{\varepsilon}\{F(t, x+\varepsilon)-F(t, x)\}
$$

We check easily that the function $H_{\varepsilon}(s, x)$ is of bounded $\gamma$-variation in $x$ uniformly in $s$ and of bounded $p, q$-variation in $(s, x)$, where $1 \leq \gamma<2$ and $p, q \geq 1,2 q+1>2 p q$ for any fixed $\varepsilon>0$ (see Proof of Theorem 1 above for similar arguments). It follows:

$$
\int_{0}^{t} \int_{\mathbb{R}} H_{\varepsilon}(s, x) d L_{s}^{x} \rightarrow \int_{0}^{t} \int_{\mathbb{R}} F(s, x) d L_{s}^{x}
$$

and

$$
\int_{0}^{t} \int_{\mathbb{R}} H_{\varepsilon}(s, x) d L_{s}^{x}=\frac{1}{\varepsilon} \int_{0}^{t}\left\{F\left(s, X_{s}\right)-F\left(s, X_{s}+\varepsilon\right)\right\} d\langle X, X\rangle_{s}
$$

\section{Occupation Time Formula}

When $F_{x}(t, x)=f(t, x)$ exists, (1.1) becomes the classical occupation time formula for continuous semimartinagles:

$$
\int_{0}^{t} f\left(s, X_{s}\right) d\langle X, X\rangle_{s}=\int_{\mathbb{R}} \int_{0}^{t} f(s, x) d_{s} L_{s}^{x} d x
$$

\section{References}

[1] C. Feng and H. Zhao, Two-parameter p, q-variation Paths and Integrations of Local Times, Potential Analysis, Vol. 25 (2006), .

[2] I. Serot, Temps local et densits d'occupation: panorama. [A survey of local time and occupation density] Ann. I.S.U.P. 46 (2002), no. 3, 21-41. 